\title{
EL SÍNDROME DE FATIGA CRÓNICA: CARACTERÍSTICAS PSICOLÓGICAS Y TERAPIA COGNITIVO-CONDUCTUAL
}

\author{
BONIFACIO SANDÍN \\ Universidad Nacional de Educación a Distancia \\ «No conozco a nadie que haya buscado tratamiento psiquiátrico, \\ porque el síndrome de fatiga crónica es una enfermedad física. \\ Yo no estoy loco, estoy enfermo" \\ A. Cherreson (Am I sick or just tired? New Women, 1991) \\ "El descanso en sí está contraindicado en el síndrome de fatiga crónica" \\ Royal Colleges of Physicians, Psychiatrists, \\ and General Practitioners (Londres, 1996)
}

\begin{abstract}
El síndrome de fatiga crónica (SFC) es una nueva entidad clínica caracterizada por la presencia de fatiga grave e incapacitante, que se da en combinación con una serie de síntomas físicos y psicológicos. El SFC aparece como categoría diagnóstica a finales de los años ochenta, es un trastorno muy poco conocido, y ha recibido escasa atención desde la psicología científica. La finalidad del presente artículo ha consistido en examinar algunos aspectos conceptuales y aplicados sobre el SFC desde una perspectiva psicopatológica y clínica. En el artículo se subraya el estado actual sobre su diagnóstico, y se describen algunos mecanismos psicológicos y estrategias de intervención cognitivo-conductual aplicables al SFC.
\end{abstract}

Palabras clave: Síndrome de fatiga crónica, SFC, terapia cognitivo-conductual, psicopatología.

Chronic fatigue syndrome: Psychological characteristics and cognitive-behavioral therapy

Chronic fatigue syndrome (CFS) is a new clinically defined condition characterized by severe disabling fatigue and a combination of physical and psychological symptoms. The CFS emerged as a diagnostic category during the late 1980 s, is a poorly understood disorder, and has received scant consideration in the psychological scientific literature. The purpose of this article was to examine several conceptual and applied issues of the CFS from a psychopathological and clinical perspective. The paper underscores the current state of diagnosis, and describes son psychological mechanisms and cognitive-behavioral interventions for CFS.

Key words: Chronic fatigue syndrome, CFS, cognitive-behavioral therapy, psychopathology.

INTRODUCCIÓN: LA FATIGA CRÓNICA COMO FENÓMENO CLÍNICO

La fatiga es un fenómeno muy común en todos los seres humanos y en todos los

Correspondencia: Bonifacio Sandín, Universidad Nacional de Educación a Distancia, Facultad de Psicología, Juan del Rosal 10 (Ciudad Universitaria), 28040 Madrid. Correo-e: bsandin@psi.uned.es Parte de este trabajo fue presentado a las I Jornadas del Síndrome de Fatiga Crónica de la Asociación Madrileña de Encefalomielitis Miálgica/Síndrome de Fatiga Crónica y Disfunción Inmune, celebrado en Madrid el 22 de noviembre de 2004. tiempos. Todos experimentamos la sensación de fatiga o cansancio de algún modo y en algún momento. Sin embargo, cuando hablamos de fatiga crónica o del síndrome de fatiga crónica, nos estamos refiriendo a un tipo de fatiga patológica, esto es, una fatiga que produce malestar y sufrimiento y que perturba la calidad de vida del individuo. Aunque la fatiga se ha asociado a muchos trastornos médicos, podríamos decir que la fatiga crónica tiene interés en sí misma por sus posibles implicaciones sobre la salud y el 
bienestar personal, ya que puede provocar importantes perturbaciones en el individuo, tales como incapacitación, pérdida de trabajo, o reducción de las actividades sociales (Sandín, 2000, 2002). Un aspecto que llama la atención es que, a pesar de ello, hasta el momento presente se haya prestado tan poca atención al estudio científico de la fatiga como fenómeno clínico. Tal vez esto se deba a que la fatiga suele ser de naturaleza inespecífica, y multietiológica, y porque suele asociarse a resultados no fatales en el individuo, además de las dificultades de medida que suele encerrar la evaluación de la propia fatiga.

El hecho de que la fatiga crónica tenga interés en sí misma, y no únicamente como un síntoma de otro trastorno, se debe también al hecho de que ésta, no sólo no es algo trivial, sino que es un fenómeno muy prevalente en la sociedad actual. Se ha sugerido que la prevalencia de la fatiga prolongada varía entre un 20 y un $40 \%$ (Kellner, 1994), pudiendo llegar a producir un grado de malestar e incapacitación similares a los asociados a otras enfermedades crónicas. Son muchos los pacientes que buscan ayuda médica debido a problemas de fatiga. La fatiga crónica se sitúa entre los problemas más comunes que se contemplan en atención primaria, junto con el dolor de garganta, los catarros, el dolor en el pecho, el dolor abdominal, los vértigos y las jaquecas (Komaroff y Fagioli, 1996).

La fatiga crónica es una queja común en los pacientes que acuden a centros clínicos, y suele asociarse a una amplia variedad de problemas de salud tanto médicos como psicológicos (alteraciones emocionales). Sin embargo, en realidad son muy pocos los estudios que han estudiado la relación entre la fatiga y tales problemas, lo cual podría deberse a la propia naturaleza de la fatiga, esto es, ser un fenómeno universal y ser de naturaleza no específica. La fatiga crónica no debe confundirse con el estado de ánimo decaído o deprimido, ni con la falta de interés. Clínicamente hablando, el diagnóstico de la fatiga crónica implica que se den los siguientes criterios (Sandín, 2000):

(1) Ser motivo de queja o preocupación.

(2) Afectar significativamente al funcionamiento de la persona.

(3) Ser desproporcionada con respecto al ejercicio efectuado.

(4) Representar un cambio claro del estado previo.

(5) Ser persistente ( $\geq$ de 6 meses), o si es intermitente debe estar presente más del 50\% del tiempo.

La fatiga prolongada suele definirse, de forma arbitraria, como aquella que tiene al menos un mes de duración. Este tipo de fatiga es bastante común en la población general, habiéndose estimado que ocurre entre el $19 \%$ y el $28 \%$ de la población (Jason et al., 1997). En aquellas personas que experimentan fatiga prolongada, si esta persiste más allá de los 6 meses, se define como fatiga crónica. Estudios recientes han puesto de relieve que la fatiga crónica (i.e., fatiga grave) es bastante menos común que la fatiga prolongada, aunque se da con bastante frecuencia en la población general. Aunque las tasas de prevalencia han variado de unos estudios a otros, empleando criterios similares a los indicados arriba, se han estimado tasas de prevalencia puntual que oscilan entre el $8 \%$ (Fukuda et al., 1997) y el $11,3 \%$ (Wessely et al., 1997).

La fatiga, por otra parte, puede estar asociada o producida por diversos factores fisiológicos o psicológicos (Komaroff y Fagioli, 1996) que, en principio, no tienen porqué poseer un carácter de naturaleza clínica. Entre estos factores fisiológicos cabrían mencionarse los siguientes: (1) el incremento del ejercicio físico, (2) 
el descanso inadecuado (p.ej., el descanso excesivo produce fatiga y alteraciones fisiológicas varias), (3) un estilo de vida sedentario (la falta de actividad induce fatiga), (4) los estresores ambientales (p.ej., ruido, calor, vibración), (5) el abuso de sustancias (p.ej., alcoholismo, abuso de cafeína) y (6) el embarazo. La fatiga crónica también puede asociarse a factores psicológicos, aunque la evidencia reciente suele indicar que se asocia fundamentalmente a diversos tipos de trastornos emocionales, entre los que se incluyen la depresión, los trastornos de ansiedad (especialmente el trastorno de pánico), y los trastornos somatoformes. Un aspecto que ha llamado la atención, y que merece la pena resaltarse, es que la fatiga crónica parece relacionarse más estrechamente con los trastornos emocionales que con las enfermedades físicas (Sandín, 2000), lo cual sugiere que estos trastornos psicológicos pueden ser causa de la fatiga de forma más acusada que las diversas enfermedades físicas.

\section{EL SÍNDROME DE FATIGA CRÓNICA (SFC): ¿UNA NUEVA ENFERMEDAD?}

Un tipo de fatiga crónica que ocurre de forma persistente y no posee una clara explicación médica se ha denominado «síndrome de fatiga crónica». Tal ha sido el impacto que ha producido en la comunidad científica la descripción de este síndrome, que no pocos autores de prestigio han resaltado y asumido que se trata de la definición de una nueva enfermedad (véase, p.ej., Jason et al., 1997). De acuerdo con estos autores, muchos médicos han tendido a minimizar la gravedad del SFC y a interpretar que el síndrome es equivalente a un trastorno mental (algo similar al antiguo trastorno conocido como "neurastenia»), lo cual ha tenido consecuencias negativas para su investigación y tratamiento.
El síndrome de fatiga crónica (SFC) es un término reciente, que aparece definido en la literatura científica por primera vez a finales de los años ochenta (en 1987, por el grupo Centers for Disease Control and Prevention of Chronic Fatigue Syndrome). A partir de esta fecha se han venido mejorando los criterios de diagnóstico de este síndrome, describiéndose los casos de exclusión correspondientes a otros trastornos (Sandín, 1999, 2004). Puesto que este síndrome no aparece descrito en el DSM-IV, ni en otros sistemas de clasificación de las enfermedades mentales, los esfuerzos realizados por este grupo han sido de enorme interés y ayuda para su delimitación nosológica y tratamiento. En 1994, este grupo llevó a cabo una revisión de las clasificaciones anteriores y llegó a un consenso sobre los nuevos criterios de diagnóstico del SFC, especificando también los criterios de exclusión, así como los procedimientos de evaluación clínica recomendados (Fukuda et al., 1994). En la Tabla 1 indicamos estos criterios, los cuales son los que se utilizan actualmente para el diagnóstico del SFC.

Tal y como se indica en la Tabla 1 , existe un primer criterio referido a la existencia de fatiga crónica inexplicada por razones médicas que debe reunir una serie de condiciones ( 5 en total). Además, deben darse al menos 4 de los ocho síntomas que se indican para el Criterio B. Todos estos síntomas deben haber ocurrido durante al menos 6 o más meses consecutivos y no deben haber precedido a la aparición de la fatiga crónica. La pérdida de memoria o de capacidad de concentración debe ser lo suficientemente grave como para producir una reducción significativa de los niveles previos de funcionamiento laboral, educativo, social o personal. Antes de esta revisión de los criterios, se incluían otros síntomas válidos para el diagnóstico del SFC, tales como la presencia de 
Tabla 1. Criterios de diagnóstico del SFC (Centers for Disease Control and Prevention of Chronic Fatigue Syndrome) (Sandín, 1999)

A. Fatiga crónica evaluada clínicamente e inexplicada médicamente (se dan los 5):

(1) Fatiga crónica persistente o que reaparece ( $\geq 6$ meses de duración)

(2) De comienzo nuevo ( no habitual)

(3) No debida al ejercicio

(4) No se alivia o desaparece con el descanso

(5) Produce deterioro de las actividades personales, sociales, educativas o laborales

B. Al menos se dan 4 de los siguientes síntomas ( $\geq 6$ meses de duración):

(1) Pérdida grave de memoria o de capacidad de concentración

(2) Dolor de garganta

(3) Nódulos linfáticos sensibles (cervicales o en axilas)

(4) Dolor muscular

(5) Dolor en articulaciones (sin hinchazón o enrojecimiento)

(6) Dolor de cabeza nuevo

(7) Sueño no reparador

(8) Malestar tras ejercicio (durante más de 24 horas)

C. No puede diagnosticarse SFC si se da alguna de las siguientes condiciones:

(1) Cualquier enfermedad médica activa que pueda explicar la fatiga crónica (p.ej., hipertiroidismo no tratado).

(2) Cualquier enfermedad médica diagnosticada previamente, cuya curación aún no ha sido suficientemente resuelta, y cuya actividad puede explicar la fatiga crónica (p.ej., casos de hepatitis B o C tratados previamente).

(3) Cualquiera de los siguientes trastornos mentales: Depresión mayor psicótica, trastorno del estado de ánimo bipolar, esquizofrenia, trastornos delirantes, demencia, anorexia nerviosa, o bulimia nerviosa.

(4) Abuso de alcohol u otras sustancias durante los dos años anteriores al comienzo de la fatiga crónica y en cualquier tiempo posterior.

(5) Obesidad grave, definida por el índice de masa corporal (IMC) $\geq 45$ [IMC = peso en $\mathrm{kg} /$ (altura en metros) $\left.)^{2}\right]$

Nota: Elaborado a partir de Fukuda et al. (1994).

febrícula (entre $37,5^{\circ}$ y $38,6^{\circ}$ de temperatura), los resfriados, debilidad muscular en general, fatiga prolongada tras el ejercicio (más de 24 horas), trastornos del sueño, quejas neuropsicológicas (p.ej., fobia a la luz, irritabilidad, confusión), etc. Los casos de fatiga crónica evaluada clínicamente e inexplicada médicamente que no reúnan al menos 4 de los síntomas indicados en la tabla son considerados como casos de fatiga crónica idiopática. Por tanto, pueden darse casos que experimenten fatiga crónica y únicamente 3 síntomas entre los 8 referidos en la tabla, aunque también exhiban otros síntomas como los indicados arriba (febrícula, etc.). Estos casos no podrí- an ser diagnosticados de SFC, aunque sí de fatiga crónica idiopática. Aunque es necesario establecer limites precisos en todo diagnóstico, es probable que, en algunos casos, existan muy pocas diferencias entre un caso de SFC y otro de fatiga crónica idiopática. Los estudios epidemiológicos sugieren que el SFC es menos prevalente que la fatiga crónica idiopática, habiéndose referido datos que sugieren una prevalencia puntual del SFC que oscila entre el 2,8\% (Fukuda et al., 1997) y el $2,6 \%$ (Wessely et al., 1997), lo que sugiere que la prevalencia puntual de la fatiga crónica idiopática podría variar entre el $5,2 \%$ y el $8,7 \%$ (véanse los datos sobre prevalencia pun- 
tual de la fatiga crónica inexplicada indicados arriba).

Si bien la inclusión de la categoría de fatiga crónica idiopática ha sido uno de los aspectos más controvertidos de la clasificación de Fukuda et al. (1994), una aportación importante de esta nueva categorización es sin duda el establecimiento de las condiciones de exclusión e inclusión para el diagnóstico del SFC. En la Tabla 1 indicamos las condiciones generales de exclusión (referido en la tabla como Criterio C). Siempre que en el paciente ocurra alguna de estas condiciones, no podrá establecerse el diagnóstico de SFC. En cuanto a las condiciones de inclusión, se citan las siguientes: fibromialgia, trastornos de ansiedad, trastornos somatoformes, depresión no psicótica o no melancólica, neurastenia, y trastorno de sensibilidad química múltiple. Esto quiere decir que puede diagnosticarse el SFC junto con cualquiera de estas condiciones (cabrían, por tanto, diagnósticos comórbidos). Tampoco son condiciones de exclusión la existencia de enfermedades adecuadamente tratadas (p.ej., hipotiroidismo tratado con terapia hormonal sustitutiva, o asma cuyo tratamiento haya sido determinado de la función pulmonar u otras pruebas), ciertas enfermedades (p.ej., sífilis o enfermedad de Lyme) tratadas correctamente antes de aparición de la enfermedad crónica, o resultados aislados de laboratorio (p.ej., incremento de anticuerpos).

\section{¿SON LAS CAUSAS LO MISMO QUE EL MANTENIMIENTO DEL TRASTORNO?}

Las causas del SFC, al igual que de la fatiga crónica en general, pueden ser muy diversas (la etiología de este trastorno es claramente multifactorial). Como ocurre con muchos otros trastornos, existen tanto factores de predisposición como facto- res precipitantes. Entre los factores de predisposición se encuentran los trastornos psicológicos (p.ej., los trastornos de ansiedad, la depresión y los trastornos somatoformes), la alergia, la predisposición genética y familiar, y ciertas características de personalidad. Los factores precipitantes pueden ser tanto de naturaleza física, como ciertas infecciones $u$ operaciones quirúrgicas, como de naturaleza psicológica (p.ej., el estrés psicosocial). Una vez que se ha desarrollado el SFC, se han observado diversos mecanismos psicobiológicos etiopatogénicos que podrían relacionarse con la sintomatología del síndrome, fundamentalmente un deterioro en la capacidad de respuesta del sistema hipotálamo-hipófiso-corticosuprarrenal, alteración que puede causar otras perturbaciones comúnmente observadas, como un incremento de la activación inmunológica y cierta disregulación del proceso del sueño (fundamentalmente reducción del periodo de ondas delta) (Cleare, 2003; Sandín, 1999).

Es cierto que la etiología del SFC es poco conocida y que esta puede ser muy variada (p.ej., puede deberse tanto a un periodo de estrés como a una enfermedad infecciosa). Sin embargo, un fenómeno que ha llamado la atención en la literatura reciente sobre este síndrome es que muchos de los pacientes con SFC presentan elevada comorbilidad de trastornos psicológicos, especialmente depresión y trastornos de ansiedad (los trastornos psicológicos pueden ser causa o consecuencia del SFC); veces, el SFC puede confundirse con un trastorno depresivo (Moss-Morris y Petrie, 2001), ya que la fatiga es un fenómeno común durante la depresión. A partir de estas y otras observaciones parece claro que el SFC tiene una relación muy estrecha con los procesos psicológicos. Esto no quiere decir que el origen del SFC sea una causa psicológica (aunque pueda serlo o no), sino que en la manifestación clínica del 
síndrome desempeñan un papel importante los fenómenos psicológicos. En muchas enfermedades médicas, aunque estas sean más o menos complejas, las causas pueden explicarse a partir de un modelo simple (p.ej., como ocurre en una enfermedad infecciosa). Sin embargo, el SFC no puede explicarse de forma simple, ya que el elemento que precipita el SFC puede no ser el mismo que el que lo mantiene, por lo que el tratamiento no debe estar basado en la naturaleza del agente causal.

Una vez que se ha desarrollado el SFC este adquiere cierta autonomía que obedece a un conjunto de mecanismos psicológicos, más que médicos. Incluso, con no poca frecuencia los médicos se han equivocado al aconsejar a estos pacientes descanso prolongado, por considerar que se trata de un problema estrictamente médico (como si se tratase de algo similar una infección viral), cuando en realidad el descanso en cama prolongado debe prescribirse con suma cautela, ya que el paciente puede quedar atrapado en un circulo vicioso de inmovilidad y descondicionamiento (debilidad muscular). El descanso prolongado (o mejor la inactividad) produce, entre otras cosas, descondicionamiento neuromuscular, el cual se asocia a dolor muscular tras mínimo ejercicio (también incrementa la sensación de esfuerzo tras el ejercicio), pérdida de masa muscular y pérdida generalizada de la forma física (se altera la histología muscular). El paciente con SFC, sea cual sea la causa, suele quedar encerrado en un círculo vicioso de inactividad que le lleva al desastre.

Son varios los autores que han venido alertando sobre este mecanismo de autoperpetuación que se da en el SFC (Sharpe et al., 1997; Wessely et., 1999). Estos autores han sugerido que el SFC puede estar producido por múltiples causas, incluido el caso de haber padecido una enfermedad grave (p.ej., por una infec- ción viral). Sin embargo, una vez ocurrido, el SFC se perpetúa por un proceso psicopatológico que implica la evitación del ejercicio físico. La reducción de la actividad produce en el individuo descondicionamiento físico, e incremento de la debilidad, de la sintomatología psicosomática y del malestar general. El mantenimiento del SFC implica la interrelación de diversos factores psicológicos y físicos, entre los que se incluyen los factores cognitivos y de personalidad (p.ej., creencias de enfermedad y perfeccionismo), respuestas emocionales (p.ej., ansiedad, depresión y frustración), determinados comportamientos (p.ej., evitación del ejercicio, inactividad prolongada), y manifestaciones fisiológicas o somáticas (p.ej., descondicionamiento, cambios inmunológicos, alteraciones neuroendocrinas). En el mantenimiento del SFC también juegan un papel importante los factores sociales, entre los cuales caben destacarse los refuerzos sociales y los procesos de estigmatización. Como primer paso para el tratamiento cognitivoconductual del SFC, el psicólogo debe llevar a cabo con el paciente una formulación psicológica (cognitivo-conductual) sobre el mantenimiento del síndrome. El SFC es un ejemplo claro en el que, no sólo el conocimiento psicológico de las características del paciente es importante para el tratamiento, sino que el conocimiento psicopatológico del caso es fundamental y guía para la delimitación y aplicación del protocolo de terapia cognitivo-conductual.

\section{TERAPIA COGNITIVO-CONDUCTUAL}

Las terapias farmacológicas que se han utilizado para el tratamiento del SFC (p.ej., administración antidepresivos, corticosteroides, inyecciones de magnesio, etc.) en realidad no constituyen tratamientos específicos de este síndrome 
(Friedberg y Jason, 2001), y los resultados tras su administración han sido más bien desalentadores (Reid et al., 2000). El único tratamiento que ha demostrado una eficacia significativa en los pacientes con diagnóstico de SFC es la terapia cognitivo-conductual, la cual ha adquirido un importante desarrollo en los últimos años (véase, p.ej., Friedberg y Jason, 2001; Jasón et al., 2003; Raine et al., 2002; Sharpe et al., 1997). Aunque se han desarrollado varios protocolos, en general la mayoría se basan en el ejercicio físico programado, el control y afrontamiento del estrés asociado a la enfermedad, y la reestructuración cognitiva.

El conocido método de Friedberg y Jason (2001) utiliza una combinación de terapia de habilidades de afrontamiento y control del ritmo de actividad con objeto de incrementar la funcionalidad física y la calidad de vida. Para ello controla los niveles de energía, la fatiga, y los niveles de actividad del paciente. Mediante las habilidades de afrontamiento, se enseña a los pacientes a utilizar pensamientos constructivos para adaptarse al malestar afectivo relacionado con la enfermedad (p.ej., se aborda la ira y frustración de los pacientes que intentan infructuosamente sobreponerse a las limitaciones de la enfermedad). Así por ejemplo, el paciente puede llevar a cabo auto-exigencias que le producen ira o frustración como, «Debería ser capaz de controlar esta enfermedad», o "No puedo soportar estar impedido de esta manera". Estas creencias pueden ser cuestionadas por otras alternativas como "¿Porqué debería ser capaz de controlar esta enfermedad, qué evidencia existe sobre ello?» Una vez que estas creencias son puestas en entredicho, pueden ser reemplazadas mediante afirmaciones de afrontamiento que animen hacia la tolerancia de las limitaciones asociadas a la enfermedad.

El procedimiento de tratamiento cognitivo-conductual desarrollado por el grupo de Sharpe (Deale et al., 1997, 2001; Sharpe, 1997; Sharpe et al., 1996, 1997) parte de la formulación (cognitivo-conductual) sobre el mantenimiento del SFC, y se desarrolla en varias sesiones. Este tratamiento, con algunas modificaciones, lo hemos implementado en nuestro grupo de la UNED, por lo que lo me referiré a él con un poco más de detalle. El tratamiento se lleva a cabo en las siguientes fases:

Fase 1. Evaluación y formulación. Esta es una fase esencial, ya que en ella se explica al paciente la base psicológica del mantenimiento de la enfermedad (formulación cognitivo-conductual). Es la fase de la psicoeducación, y en ella se pone de manifiesto la implicación de los componentes cognitivos, comportamentales, emocionales y sociales. Todos estos componentes deben ser evaluados en lo posible durante el curso del tratamiento. Durante esta fase se debe llegar a una «reformulación» de la enfermedad, siendo en ello importante (1) una explicación positiva de la enfermedad en términos biopsicosociales, y (2) un cambio de concepción de una enfermedad específica que puede ser curada por un médico, hacia una condición real pero reversible que puede ser modificada por el paciente con autoayuda práctica.

Fase 2. Intervención cognitivo-conductual. Constituye el aspecto central del tratamiento. Esta fase implica en primer lugar una planificación de la terapia en función de las características del paciente (todo resulta más difícil cuando el SFC se ha cronificado en exceso). Por ello, en primer término es preciso negociar con el paciente qué actividades se van a llevar a cabo. La terapia se dirige fundamentalmente a los cuatro aspectos siguientes: (1) Control de la actividad; puede ocurrir que sea necesario programar (de acuerdo con el paciente) un incremento gradual de la actividad (para los pacientes que 
tienen un nivel muy reducido de actividad física, y/o bien estabilizar las oscilaciones de los patrones de actividad (i.e., para los pacientes que presentan grandes oscilaciones en los niveles de actividad). (2) Revisar y reestructurar los pensamientos, generalmente relacionados con aspectos de la enfermedad; por ejemplo, pensamientos como «No puedo hacer nada excepto descansar» o "La actividad es peligrosa para la gente con mi enfermedad", pueden ser analizados a partir de la evidencia médica y el uso de experimentos conductuales (incrementos planificados de la actividad física dirigidos a probar estas predicciones). (3) Afrontamiento del estrés; el estrés no sólo pudo haber sido la causa principal del síndrome, sino que ahora constituye un elemento que agrava la enfermedad y dificulta el progreso del paciente, por lo que es necesario enseñarle formas de adaptativas de afrontar el estrés cotidiano, y especialmente los estresores asociados a su enfermedad (deben combinarse estrategias de afrontamiento cognitivas, conductuales y basadas en la relajación).

Fase 3. Revisión y modificación de actitudes desadaptativas. Los pacientes con SFC suelen poseer elevados niveles de perfeccionismo y metas poco realistas (elevadas expectativas de logro), así como otras actitudes desadaptativas que le llevan habitualmente a realizar grandes oscilaciones en su actividad cotidiana. Estas actitudes son grandes impedimentos para el progreso gradual de la rehabilitación. El paciente debe ser animado a revisar las ventajas y desventajas que pueden derivarse del hecho de mantener estas actitudes. Aunque su cambio es más difícil que el de los pensamientos desadaptativos, la discusión con el paciente puede ayudar a minimizar su influencia y a cierta modificación de las mismas.

Fase 4. Solución de problemas relacionados con dificultades prácticas. A medida que avanza la terapia y el paciente mejora, a éste pueden surgirle diversos tipos de problemas prácticos. Así por ejemplo, a un paciente que haya estado discapacitado por algún tiempo, puede resultarle difícil volver al trabajo. En algunos casos, el paciente puede decidir cambiar de trabajo (p.ej., si el trabajo anterior fue en gran parte la causa del síndrome). El terapeuta debe ayudar al paciente a resolver estos problemas.

Fase 5. Consolidación y planificación futura. Una vez finalizada la terapia, es importante evitar las recaídas. Si el terapeuta ha enseñado al paciente las estrategias adecuadas para afrontar el estrés y las variables que estaban asociadas al mantenimiento del SFC, es muy probable que no sean necesarias muchas más cosas. No obstante, siempre son útiles algunas orientaciones prácticas para prevenir futuras recaídas. En este sentido, el terapeuta debería entregar al paciente algún documento escrito en el que figurase la formulación cognitivo-conductual del SFC, así como también una relación de estrategias de afrontamiento para poner en práctica ante posibles dificultades futuras.

\section{COMENTARIOS FINALES}

Es posible que el SFC siga siendo aún el gran desconocido para muchos, por no decir la mayoría, de los profesionales que trabajan en el campo de la salud (tanto física como mental). Sin embargo, aunque la medicina no ha proporcionado información relevante sobre los mecanismos etiopatogénicos de este trastorno, ni tampoco tratamientos eficaces para abordarlo, la evidencia acumulada durante estos últimos años sobre los mecanismos que mantienen el síndrome, así como también sobre la eficacia de la terapia cognitivo-conductual, permite albergar serias y esperanzadoras expectativas 
sobre la ayuda que puedan aportar la psicología y la psiquiatría al conocimiento y al tratamiento de este trastorno. El gran esfuerzo que en estos momentos se está haciendo desde los distintos campos y especialidades de la salud (véase, Jason et al., 2003), sin duda, nos permitirán conocer los mecanismos etiopatogénicos del SFC y, en último término, nos posibilitarán desarrollar tratamientos más eficaces y efectivos de este trastorno. Algunos problemas no exentos de relevancia como la delimitación de posibles subtipos de SFC (p.ej., según la mayor o menor preponderancia de ciertos síntomas), las características de comorbilidad (p.ej., distinción entre SFC puro y SFC con elevada comorbilidad de trastornos psicológicos), la relación con la depresión, el solapamiento con otros síndromes cercanos (p.ej., el síndrome de fibromialgia), o la separación y/o similitud de la denominada "fatiga crónica idiopática», constituyen retos futuros cuya resolución necesariamente redundará en un mejor conocimiento y tratamiento del SFC.

\section{REFERENCIAS BIBLIOGRÁFICAS}

Cleare, A.J. (2003). Neuroendocrine dysfunction. En L.A. Jason, P.A. Fennell y R.R. Taylor (Eds.), Handbook of chronic fatigue syndrome (pp. 331-360). Hoboken, NJ: Wiley.

Deale, A., Chalder, T., Marks, I., y Wessely, S. (1997). Cognitive behavior therapy for chronic fatigue syndrome: A randomised controlled trial. American Journal of Psychiatry, 154, 408-414.

Deale, A., Husain, K., Chalder, T., y Wessely, S. (2001). Long-term outcome of cognitive behavior therapy versus relaxation therapy for chronic fatigue syndrome: A 5-year follow-up study. American Journal of Psychiatry, 158, 2038-2042.

Friedberg, F., y Jason, L.A. (1998). Understanding chronic fatigue syndrome. Was- hington, DC: American Psychological Association.

Friedberg, F., y Jason, L.A. (2001). Chronic fatigue syndrome and fibromyalgia: Clinical assessment and treatment. Journal of Clinical Psychology, 57, 433-455.

Fukuda, K., Dobbins, J.G., Wilson, L.J., Dunn, R.A., Willcox, K., y Woods, D.S. (1997). An epidemiologic study of fatigue with relevance for the chronic fatigue syndrome. Journal of Psychiatric Research, 31, 19-29. Fukuda, K., Straus, S.E., Hickie, I., Sharpe, M.C., Dobbins, J.G., Komaroff, A., y el International Chronic Fatigue Syndrome Study Group (1994). The chronic fatigue syndrome: A comprehensive approach to its definition and study. Annals of International Medicine, 121, 953-959.

Jason, L.A. Fennell, P.A., y Taylor, R.R. (Eds.) (2003). Handbook of chronic fatigue syndrome. Hoboken, NJ: Wiley.

Jason, L.A., Richman, J.A., Friedberg, F., Wagner, L., Taylor, R., y Jordan, K.M. (1997). Politics, science, and the mergence of a new disease. American Psychologist, 52, 973-983.

Kellner, R. (1994). Psychosomatic syndromes, somatization and somatoform disorders. Psychotherapy and Psychosomatics, 61, 4-24.

Komaroff, A.L., y Fagioli, L. (1996). Medical assessment of fatigue and chronic fatigue syndrome. En M.A. Demitrack y S.E. Abbey (Eds.), Chronic fatigue syndrome: An integrative approach to evaluation and treatment (pp. 154-181). New York: Guilford Press.

Moss-Morris, R., y Petrie, K.J. (2001). Discriminating between chronic fatigue syndrome and depression: A cognitive analysis. Psychological Medicine, 31, 469-479.

Raine, H., Haines, A., y Sensky, T. (2002). Systematic review of mental health interventions for patients with common somatic symptoms: Can research evidence form secondary care be extrapolated to primary care? British Medical Journal, 325, 10821093.

Sandín, B. (1999). El sindrome de fatiga cronica. Conferencia. $X$ Cursos de Verano de la UNED. Ávila, 12-16 de julio.

Sandín, B. (2000). Síndrome de fatiga crónica. Madrid: UNED (policopiado) 
Sandín, B. (2002). Papel de las emociones negativas en el trastorno cardiovascular: Un análisis crítico. Revista de Psicopatología y Psicología Clínica, 7, 1-18.

Sandín, B. (2004). Tratamiento del síndrome de fatiga crónica. Conferencia. Cursos de Postgrado en Psicopatología y Salud de la UNED. Madrid, 21 de febrero.

Sharpe, M. (1997). Cognitive behavior therapy for functional somatic complaints: The example of chronic fatigue syndrome. Psychosomatics, 38, 356-362.

Sharpe, M., Chalder, T., Palmer, I., y Wessely, S. (1997). Chronic fatigue syndrome: A practical guide to assessment and management. General Hospital Psychiatry, 19, 185-199.
Sharpe, M., Hawton, K.E., Simkin, S., Surawy, C., Klimes, I., Peto, T.E.A., Warrell, D., y Seagroat, V. (1996). Cognitive therapy for chronic fatigue syndrome: $A$ randomised controlled clinical trial. British Medical Journal, 312, 22-26.

Wessely, S., Chalder, T., Hirsch, S., Wallace, P., y Wright, D. (1997). The prevalence and morbidity of chronic fatigue and chronic fatigue syndrome: A prospective primary care study. American Journal of Public Health, 87, 1449-1455.

Wessely, S., Hotopf, M., y Sharpe, M. (1999). Chronic fatigue and its syndromes. Oxford: Oxford University Press. 